\title{
Camouflage Technique Based Multifunctional Army Robot
}

\author{
Rashmi Hegde $^{1 *}$, G. T. Raju ${ }^{2}$ \\ ${ }^{I}$ M.Tech. Student, Dept. of Computer Science and Engineering, RNS Institute of Technology, Bengaluru, India \\ ${ }^{2}$ Professor \& HoD, Dept. of Computer Science and Engineering, RNS Institute of Technology, Bengaluru, India \\ *Corresponding author: rashmihegdeinchara@gmail.com
}

\begin{abstract}
These days, numerous costs are made inside the field of safeguard in receiving crude safety efforts to monitor the fringe from the trespassers. Some military associations take the help of robot inside the hazard inclined regions which are not that powerful when done by armed force men. These Army robots are restricting with the camera, sensors, locator and video screen. The principle goal of our framework is to ask covered including some extra parameters like Wi- Fi module for continuous information prepared by the camera at the video screen and PIR sensor to follow the interlopers. Hence, the proposed framework utilizing Wi-Fi decreases mistakes at guard and keeps the state secure from the enemy. Cover Robot assumes a significant job in sparing human loses and the harms that happen during debacles. Therefore, it will acquire significance inside the up and coming period.
\end{abstract}

Keywords: Camouflage technique, Haar cascade algorithm, Internet of Thing.

\section{Introduction}

The robot essentially comprises of a vehicle mounted with cameras and has two capabilities, one is which catches the photos and distinguishes shading in like manner as a piece of covering highlight and in this way the other one capability is the observation. As a substitution pattern we've utilized remote handset WIFI to expand the scope of correspondence among transmitter and beneficiary. The proposed robot can unobtrusively go into foe zone and send data through camera to the controller. one among the striking highlights of this robot is covering for example the camera catches the picture and along these lines the shade of the incorporating will be distinguished at the backend and predictable with that robot will change its shading. Because of this component the robot can't be handily distinguished by adversaries.

The advancement of the robot is remotely controlled through mobile phone. Since human life is typically more valuable, the proposed robot is routinely the substitution of warriors in war domains. It is animated by creatures like chameleon. The camouflage robot furthermore can be utilized in star lodgings, strip malls, enhancements showrooms, movement aides, and rescue bunches during catastrophes. On the other hand, at places like where there are routinely perils from intruders or mental oppressors.

\section{Problem Statement}

\section{A. Existing system}

Fundamentally, Army Robot is equipped for performing errands, for example, motion, detecting the hurtful gas, detecting the people underneath the surface, metal identification. Armed force robot is an independent robot involving remote camera which can be utilized as a government agent and Bluetooth used to control it remote.

Manual Boarder security: Soldier just deal with the Defense framework. Existing is sensor less catching framework dependent on raspberry pi-based controllers. It can't screen definite movement without IR sensor. It is beneficial as it offers unwavering quality and security on the two sides. We are utilizing Raspberry PI for this task. Any development in profoundly made sure about zone, for example, Border by utilizing robotization. The computerization is sensor-based framework focus on the living item inside the scope of sensor. The programmed firearm focusing on is essential base on IR sensors, camera. Up to that point, fringe is finished by Iron Spike wires, and a watch tower from which an individual persistently blazing the light over the outskirt zone day and night.

\section{B. Proposed system}

The possibility of the Army Robot depends on the cover procedures. The point of the venture is to configuration, fabricate and work by means of a Smart telephone, utilized as remote-control gadget can replicate the shading as needs be with the ground surface where it will be proceeding onward, thus being covered to the outside world.

The fact of the matter is to make an insightful observation system which can be seen by owner remotely through android application. As it is connected with the system with IOT, structure will send the message spring up to android device when an interference is perceived inside the room. It is required to make and complete and moderate simplicity web-camera based perception structure for remote security checking. Affirmed customer can access to their checking structure remotely by methods for web with the use a mobile phone and screen the situation on application. 
Volume-3, Issue-9, September-2020

\section{IJRESM journals.resaim.com/ijresm | ISSN (Online): 2581-5792 | RESAIM Publishing}

\section{Related Work}

As per our study, the explored frameworks utilized shading identification sensor which have an issue of distinguishing shading because of which had constraints over a specific range. Metal indicator and gas sensors were utilized which made the framework progressively perplexing. Those indicators and sensors are evacuated to diminish the heaviness of the robot. Additionally, the framework comprises TV tuner card which made the framework complex. So as to lessen the expense and unpredictability it is expelled. The point of designing cover is to make the identification and acknowledgment target trouble in the machine helped eye looking through objective in the large expansiveness foundation around. [3] The sensor switches every essential shading RGB, individually and checks what power of shading is reflected by the outside of location. This reflected power is changed over to 8-piece esteem. For instance, a RED surface will firmly reflect RED. While a Yellow surface will reflect RED and GREEN both. As per the enlistment rule of the three essential hues which make different hues in nature, when the estimation of three essential hues is affirmed, the shade of the tried article is known. Knowing the estimation of RGB assists individuals with picking up the shade of the light which is anticipated onto the sensor since each shading compare to just one estimation of RGB. [2] The proposed framework gives some assistance to our security powers in identification of gatecrashers. The robot can likewise be utilized in high elevation regions where human can't endure. Besides, the disguising highlight makes it hard to identify the robot by exposed natural eye. There is extension to improve the framework by arranging it with multicolor covering.

\section{A. System architecture}

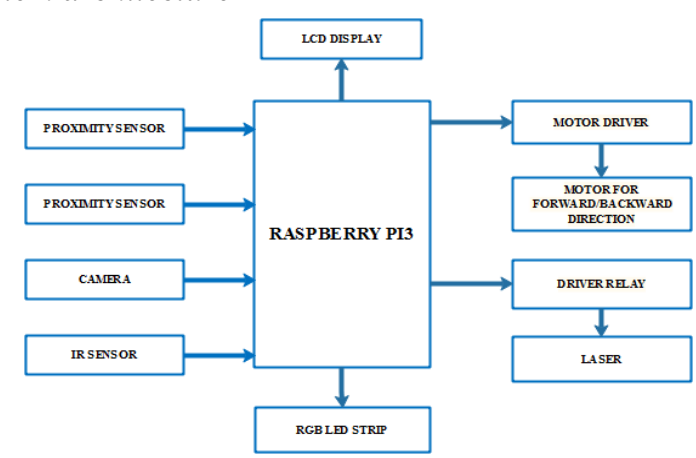

Fig 1. System architecture

This framework is intended to follow the article progressively and give security by methods for peripherals appended to this framework. The working of the framework comprises of a few stages which incorporates the handling of video signal from camera. The video is first changed over into the crude advanced configuration and afterward its luminance part is extricated by applying picture preparing calculations. At that point each edge is contrasted and the past casing so as to identify the movement in the present casing. It is finished by utilizing foundation deduction strategy. In this work, we applied scaling and smoothing methods to evacuate clamor and to improve the sharpness of the picture. At that point we determined the distinction picture by taking away the foundation data. At that point the resultant crude double picture is additionally handled by utilizing morphological tasks which brings about the identification of different items. At that point we applied mass examination to interface all pieces of the distinguished picture. The following stage was to decide the directions of the chose target which is accomplished by sending the data to the miniaturized scale controller-based framework which thinks about the directions of the present casing with the past one. Miniaturized scale controller at that point performs two capacities; one is to enact the fringe gadgets and the second is to take the choice so as to move the firearm to the ideal area. When the objective is chosen, the microcontroller controls the development of the firearm by utilizing the stepper engine. After the article is followed, the choice to shoot the objective is accomplished physically or naturally by utilizing microcontroller based.

\section{B. Methodologies}

On robot side both the Input and Output is completed whereas on PC side all the image processing is completed. Robot has of these input devices like color sensing camera, video feeding camera and obstacle sensor to gather all the specified data for processing. This data is then transmitted wirelessly to $\mathrm{PC}$ via Wi-Fi trans-receiver. Computer then does the processing of the received data using various algorithms for image processing. It determines the colour of background and transmits this data to robot. Through PC the user also can provide the movement commands to the robot. One among the important functions of PC is to display the live video feed received from the robot. All the transmission is completed serially using Wi-Fi trans- receiver. Robot can output the received color by changing the colour of LEDs covering the chasse. this is often done by turning on one among the three relays present on the robot. The PC using which we'll be ready to handle the movement of the robot.

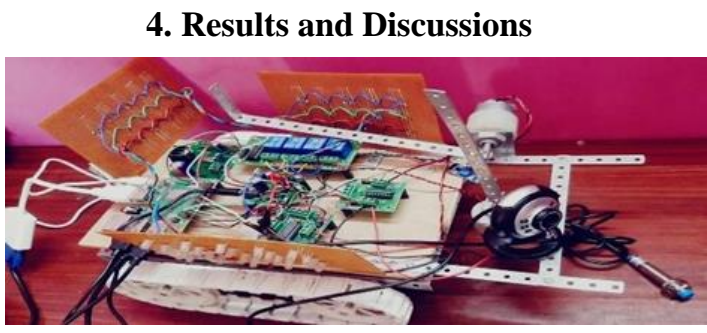

Fig. 2. Camouflage army robot

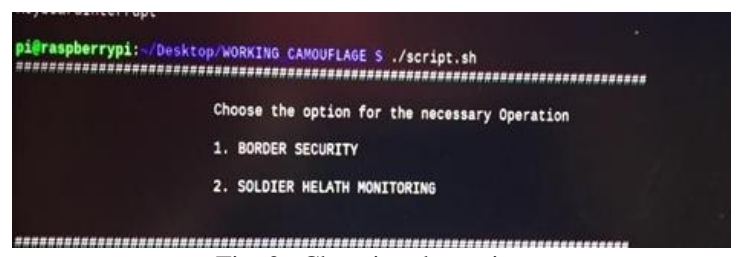

Fig. 3. Choosing the option 
Volume-3, Issue-9, September-2020

\section{IJRESM journals.resaim.com/ijresm | ISSN (Online): 2581-5792 | RESAIM Publishing}

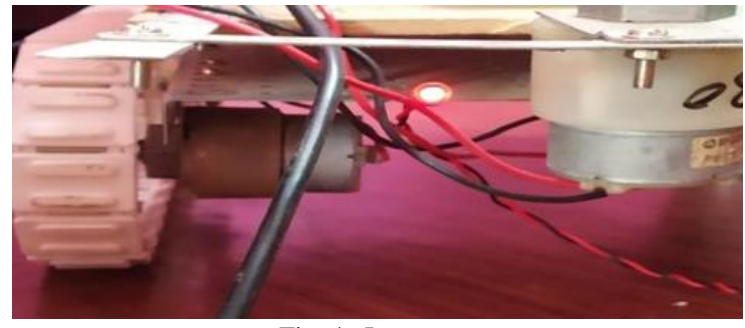

Fig. 4. Laser gun

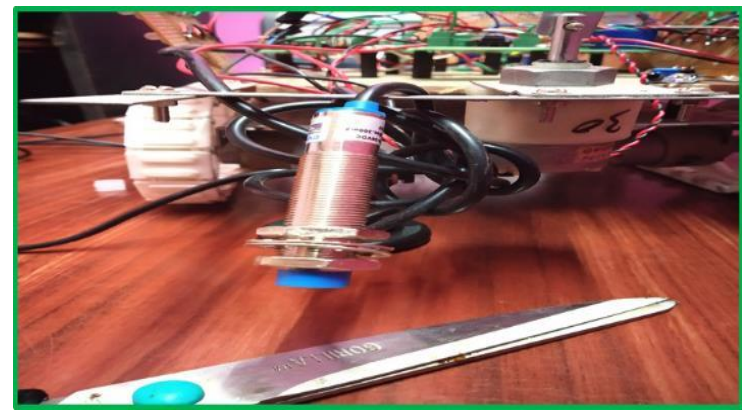

Fig. 5. Metal detection

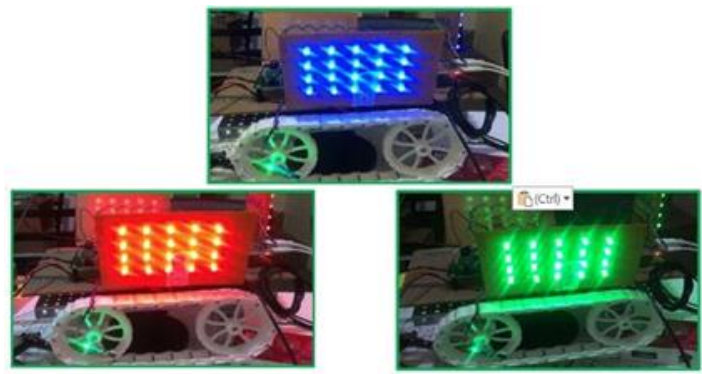

Fig. 6. Camouflage technique

\section{Conclusion}

The proposed framework is a substitution to human life. As Human life is usually more prioritize this proposed robot helps to act as a security system and also a life savior. It enacts and plays a crucial role of keeping eye on the war field areas and captures the environment. As it is predicated on the Chameleons color changing effect, the robot changes its color consistent with the encompassing environment and is hidden from the enemy's insight. Robot to robot communication enables operation in out of coverage area. In addition, the disguising highlight makes it hard to recognize the robot by exposed natural eye. In this way, in all the proposed framework gives some assistance to our security powers in location of interlopers. The robot can likewise be used in high height zones where human cannot endure.

\section{References}

[1] Cristina Cică, Lucian Filipoaia, "Sustainability enabling research methods for development of integrated border security systems."

[2] Mosad Khatami, Lubna Alazzawi, AliElkateeb, "Large scale border security systems modeling and simulation with OPNET."

[3] Ross S. Eaton, Stan German, Arjuna Balasuriya, "Maritime Border Security using Sensors, Processing, and Platforms to Detect Dark Vessels."

[4] Axel Weissenfeld. Andreas Zoufal. Andreas Zoufal Bernhard Strobl Gustavo Fernández Domínguez, "Towards Mobile Contactless 4Fingerprint Authentication for Border Control."

[5] Ajay S. Mirchapure, "Automatic Gun Targeting System," IJAR.

[6] Rodabasannavar Sharanabasappa, Shilpa Patil, Priti Chandagad, Ms. Anuja Pati, "Automatic target detection using PIR sensors," IJRTER, 2018.

[7] N. Jayanthi, S. Indu, "Comparison of Image Matching Techniques."

[8] R. Padilla, C. F. F. Costa Filho and M. G. F. Costa, "Evaluation of Haar Cascade Classifiers Designed for Face Detection," International Journal of Computer, Electrical, Automation, Control and Information Engineering, vol. 6, no. 4, 2012.

[9] K. Visakha and S. S. Prakash, "Detection and Tracking of Human Beings in a Video Using Haar Classifier," 2018 International Conference on Inventive Research in Computing Applications (ICIRCA), Coimbatore, 2018, pp. 1-4.

[10] Yadnika Warang, Tejali Mahadik, Supriya Ojha, Asha Rawat, "Camouflage Robot-A Color Changing Spy Robot," vol. 3, no. 2, IJARIIE, 2017.

[11] Hitesh Shinde, Kirti Sonawane, Pranit Rane, Atharva Pathak, Sumita Chandak, "Camouflage Color Changing Robot," IJRASET, 2018.

[12] Ghanem Osman Elhaj Abdalla, T. Veeramanikandasamy, "Implementation of Spy Robot for a Surveillance System using Internet Protocol of Raspberry Pi." 